Papers from Italian workers in Parma and Turin show that these peptides have a higher vasodilator activity per unit weight than any other known biological substance. Hypotension is caused by direct peripheral vasodilatation particularly in the musculo-cutaneous circulation. During hypotension sympathetic reflexes increase heart rate, stroke volume and cardiac output.

Bradykinin is believed to play a physiological part in the vasodilatation which accompanies glandular activity and inflammation. Papers from England and the United States discuss the microcirculatory effects of bradykinin. There are a number of papers on kinin formation in pathological states in man, notably pancreatitis, strangulation of the gut and the carcinoid syndrome. Fairly convincing evidence is presented by Drs. Oates and Melmon from the United States to show that adrenaline acts on carcinoid tumour metastases to liberate the enzyme kallikrein into the blood. This acts on kininogen to liberate lysyl-bradykinin which is converted to bradykinin by aminopeptidase in plasma.

Evidence is slowly accumulating that these peptides have a wide range of important pharmacological actions. Although many of them share the property of causing hypotension by bringing about vasodilatation, the title of this book is misleading concerning both the scope of the papers it contains and the diversity of actions of kinins.

C. T. DOLLERY

\section{IN SEARCH OF GAS EQUATIONS}

\section{Kinetic Equations of Gases and Plasmas}

By Ta-You Wu. (Addison-Wesley Series in Advanced Physics.) Pp. vi +298. (Reading, Mass., and London: Addison-Wesley Publishing Company, 1966.) 94s.

Is recent years numerous attempts have been made to develop a kinetic theory of dense gases and plasmas, by applying the ideas of statistical mechanics to nonequilibrium processes. These attempts have had only limited success, partly because they depend on multibody collisions, partly because electrostatic forces (and other intermolecular forces to a lesser extent) decrease relatively slowly with increasing distance, and partly because the velocities of colliding molecules may not be uncorrelated. The first of these implies that the expressions for the various gas coefficients cannot be evaluated explicitly; the second and third imply that these expressions are frequently given in terms of non-convergent expansions.

Professor Wu's book gives an account of such attempts, largely stemming from the work of Bogoliubov (1946). 'The book's title is significant; it is not about gases and plasmas as such, but about mathematical methods invoked to construct their kinetic equations. Only at one point (page 154) is a comparison with experiment mentioned. This is only partly a result of difficulties in treating multibody collisions. Often the highest achievement of one of the approaches described appears to be that it supplies some measure of justification for a crude simplifying assumption made earlier on general grounds, or gives results consistent with those obtained by another of the approaches.

I found the book alternately exciting and exasperating; exciting in its discussion of basic problems like irreversibility and difficulties of convergence; exasperating in its preoccupation with equations as such, in its reference away to original papers just when one expected the keystone of an argument, and in occasional assumptions of more knowledge than could be derived from the book alone. (There is also a not inconsiderable number of misprints.) The book can fairly be regarded as an interim report. A final version, one feels, should unify the different approaches and lead to a comparison with experiment.

\section{ABSORBING TOPIC}

Mössbauer Effect Methodology

Edited by Irwin J. Gruverman. Vol. 2. (Proceedings of the 2nd Symposium, New York City, Jan. 25, 1966.) Pp. viii +191 . (New York : Plenum Press, 1966.) $\$ 12.50$.

Two meetings were held in New York, in January 1965 and January 1966, under the somewhat unattractive title of Symposium on Mössbauer Effect Methodology. Resulting from each of these, a volume of Proceedings has appeared, both of which are of substantial value to practitioners in this field. It is interesting to note that both meetings were sponsored by two commercial organizations, the New England Nuclear Corporation and the Technical Measurement Corporation.

The first volume, the subject of an earlier review, largely restricted its attention to experimental procedures and apparatus, while the second is divided into two sections with different objects.

The first section is the one which most readers will find the more useful. It consists of articles concerning the technique of analysing the raw data of Mössbauer experiments in various chemical and physical applications. For example, the analysis of recoilless fraction measurements in terms of lattice dynamics is discussed, together with the possibility of extracting the parameters of the electric field gradient tensor from Mössbauer resonances split by quadrupole interactions. There are also articles on Mössbauer measurements of non-metallic materials in the paramagnetic state and on the determination of local moments in dilute alloys of iron in diamagnetic host metals.

The second section of the volume concerns applications of the Mössbauer effect to the study of specific and especially interesting materials, such as meteoric iron minerals, haemoglobin and very thin films of iron.

Applications of the Mössbauer effect are now recorded in an extremely wide range of the journals of chemistry and physics, and the appearance of a further collection of authoritative articles between two covers will please many workers who are having difficulty in coping with the Mössbauer literature explosion.

R. E. Meads

\section{OBITUARIES}

\section{J. Robert Oppenheimer}

OPPENHEIMER was one of the most influential physicists in the middle of this century. This influence stemmed from his personality and intellectual breadth, which, when added to his very considerable scientific talents, made him a leader of the scientific community. After his very early work, his interest in physics turned increasingly toward fundamental questions of quantum electrodynamics, positron theory, cosmic ray showers, meson theory, and nuclear processes.

Oppenheimer's education was completed after the events of 1925-26 which are associated with the great names of de Broglie, Heisenberg, Pauli, Schrödinger and Dirac, and therefore he cannot be counted among these major contributors to the basic structure of quantum mechanics. Nevertheless, he very soon acquired a complete mastery of these new tools and was among the first to bring this knowledge and skill back to the United States from Cambridge and Göttingen and Zurich where he had worked. This knowledge and skill, combined with a fascinating personality, made it possible for him to establish the very important school of theoretical physics centered in Berkeley. There, and in Pasadena, California, he had close contacts with experimental physicists. The 